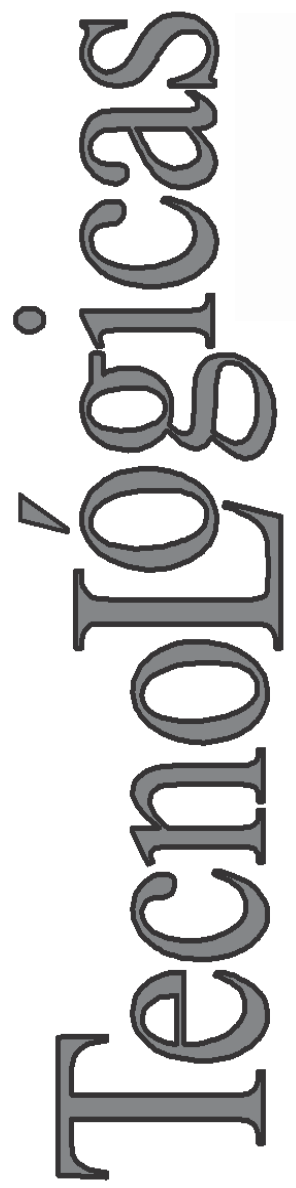

Artículo de Investigación/Research Article

\title{
Cambios en la Vitamina C y el Color durante la Cocción del Pimentón Verde (Capsicum Annuum L)
}

\section{Changes in Vitamin $C$ and Color during Cooking of Green Peppers (Capsicum Annuum L)}

\author{
Francia E. Quipo-Muñoz ${ }^{1}$ \\ Ángela M. Ramírez-Muñoz ${ }^{2}$ \\ Julián A. Rojas-Pérez ${ }^{3}$ \\ Luis E. Ordoñez-Santos ${ }^{4}$
}

Recibido: 25 de mayo de 2013 Aceptado: 11 de agosto de 2013

1 Estudiante de Ingeniería Agroindustrial, Departamento de Ingeniería, Facultad de Ingeniería y Administración, Universidad Nacional de Colombia,

Palmira-Colombia fequipom@unal.edu.co

2 Estudiante de Ingeniería Agroindustrial, Departamento de Ingeniería, Facultad de Ingeniería y Administración, Universidad Nacional de Colombia,

Palmira-Colombia anmramirezmu@unal.edu.co

3 Estudiante de Ingeniería Agroindustrial, Departamento de Ingeniería, Facultad de Ingeniería y Administración, Universidad Nacional de Colombia, Palmira-Colombia jarojaspe@unal.edu.co

4 PhD. en Ciencias Biológicas, Departamento de Ingeniería, Facultad de Ingeniería y Administración, Universidad Nacional de Colombia, Palmira-Colombia leordonezs@unal.edu.co 


\title{
Quipo et al. / Cambios en la Vitamina C y el Color durante la Cocción del Pimentón Verde (Capsicum Annuum L)
}

\section{Resumen}

La calidad del pimentón es valorada principalmente por el sabor, el color, y el contenido de vitamina C. En este estudio se exploró el efecto de la cocción en las características fisicoquímicas de los frutos de pimentón verde. Se pesaron $150 \mathrm{~g}$ cortados en tamaños de $2,2 \mathrm{~cm}$ de largo y $1,3 \mathrm{~cm}$ de ancho y se sometieron a los tratamientos térmicos (vapor y agua durante 5 min, y microondas $760 \mathrm{~W}$ durante $2 \mathrm{~min}$ ), se enfriaron rápidamente y se procedió a determinar el $\mathrm{pH}$ y acidez, concentración de vitamina $\mathrm{C}$ por el método Indofenol, contenido de clorofila total por espectrofotometría a $652 \mathrm{~nm}$ y las coordenadas de color superficial. Después de los tratamientos térmicos, la concentración de vitamina $\mathrm{C}$, el contenido de clorofila y la acidez presentaron diferencias significativas $(\mathrm{p}<0,05)$, por el contrario $\mathrm{pH}, \mathrm{L}^{*}, \mathrm{a}^{*}, \mathrm{~b}^{*}, \mathrm{C}^{*} \mathrm{y} \mathrm{h}^{\circ}$ no se vieron afecta-

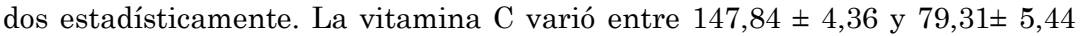
$\mathrm{mg}$ de ácido ascórbico/100 g de pimentón. La clorofila total oscilo entre 4,44 \pm 0,04 y $2,61 \pm 0,04 \mathrm{mg}$ de clorofila/100 g de pimentón. En la acidez se registraron valores entre $0,10 \pm 0,005$ y $0,07 \pm 0,001 \%$ ácido cítrico. El método de cocción por microondas obtiene el mayor nivel de retención de vitamina $\mathrm{C}$, y el color superficial del pimentón verde no es afectado por los diferentes métodos de cocción evaluados en este estudio.

\section{Palabras clave}

Antioxidante, ácido ascórbico, clorofila, CIELab, pH.

\begin{abstract}
The quality of pepper is mainly valued for flavor, color, and vitamin C. This study explored the effect of cooking on the physicochemical characteristics of the fruits of green pepper. Weighed $150 \mathrm{~g}$, cut to size of 2.2 $\mathrm{cm}$ long and $1.3 \mathrm{~cm}$ wide and subjected to heat treatment (steam and water for $5 \mathrm{~min}$, and $760 \mathrm{~W}$ microwave for $2 \mathrm{~min}$ ), and quenched proceeded to determine the $\mathrm{pH}$ and acidity, vitamin $\mathrm{C}$ concentration by the indophenol method, total chlorophyll content by spectrophotometry at $652 \mathrm{~nm}$ and the surface color coordinates. After heat treatment the concentration of vitamin $\mathrm{C}$, chlorophyll content and acidity significant differences $(\mathrm{p}<0.05)$, in contrast $\mathrm{pH}, \mathrm{L} * \mathrm{a} * \mathrm{~b}$ ${ }^{*}, \mathrm{C} *$ and $\mathrm{h}{ }^{\circ}$ not statistically affected. Vitamin $\mathrm{C}$ ranged from $147.84 \pm 4.36$ and $79.31 \pm 5.44 \mathrm{mg}$ ascorbic acid/100 $\mathrm{g}$ fresh weight. The total chlorophyll ranged from $4.44 \pm 0.04$ and $2.61 \pm 0.04 \mathrm{mg} / 100$ of pepper. In the acidity values were between $0.10 \pm 0.005$ and $0.07 \pm 0.001 \%$ citric acid. The microwave cooking method obtains the highest level of retention of vitamin $\mathrm{C}$, and the surface color of green pepper is not affected by the different cooking methods evaluated in this study.
\end{abstract}

\section{Keywords}

Antioxidant, ascorbic acid, chlorophyll, CIELab, pH. 


\section{INTRODUCCIÓN}

El pimentón (Capsicum annuum L.) es un importante cultivo agrícola en todo el mundo, no solo por ser una especie que imparte sabor, aroma, y color a los alimentos sino también por ser una fuente importante de antioxidante como el ácido ascórbico, compuestos fenólicos, flavonoides y carotenoides (Ghasemnezhad et al., 2011, Zhuang et al., 2012). Agroindustrialmente, el pimentón se utiliza para producir productos deshidratados como la paprika, los pimientos en vinagre y en rodajas o en cubitos congelados para utilizar en pizzas o en la extracción de oleorresinas. La obtención de este tipo derivados del pimentón demandan realizar operaciones de tratamientos térmicos que inactiven los complejos enzimáticos con el fin de extender la vida útil de los productos procesados del pimentón, sin embargo, los tratamientos de calor desencadenan procesos de degradación térmica de los pigmentos responsables del color, y la destrucción de los compuestos nutricionales como la vitamina $\mathrm{C}$.

$\mathrm{El}$ efecto de la cocción sobre la concentración de vitamina $\mathrm{C}$ y el color en el pimentón ha sido abordado previamente por Castro et al. (2008), Chuah et al. (2008), Ornelas-Paz et al. (2010), Castro et al. (2011), Ornelas-Paz et al. (2013) sin embargo en Colombia, a pesar de la importancia que adquiere cada día más este tipo de cultivo por sus múltiples usos a nivel industrial y en el mercado en fresco, en la literatura científica aún no se reportan trabajos que den información sobre la incidencia de los métodos de cocción en la calidad de esta hortaliza. Por esta razón, el objetivo de este trabajo es estudiar los cambios en la concentración de vitamina $\mathrm{C}$ y del color en pimentón verde durante diferentes métodos de cocción.

\section{MATERIALES Y MÉTODOS}

En el presente trabajo se utilizó pimentón verde (Capsicum annuum) proveniente del corregimiento El Bolo, situado en la zona rural de la ciudad de Palmira en el departamento del Valle del Cauca (Colombia) cultivado a 1100 m.s.n.m y a una temperatura promedio de $23^{\circ} \mathrm{C}$. Las muestras se recolectaron y se llevaron 
inmediatamente al laboratorio de Tecnología de frutas y hortalizas, de la Universidad Nacional de Colombia, sede Palmira, para su respectivo procesamiento. Los frutos del pimentón se lavaron, se desinfectaron con una solución de 50 ppm de hipoclorito durante 10 minutos y se acondicionaron en tamaños homogéneos de 2,2 cm de largo y 1,3 cm de ancho. Muestras de $150 \mathrm{~g}$ de pimentón acondicionado se sometieron a los tratamientos térmicos con vapor saturado $\left(97^{\circ} \mathrm{C}\right.$ durante cinco minutos), agua $\left(98^{\circ} \mathrm{C}\right.$ durante cinco minutos) y microonda ( $760 \mathrm{~W}$ durante dos minutos), finalizado el tratamiento de calor las muestras se enfriaron rápidamente con agua a $5^{\circ} \mathrm{C}$ e inmediatamente se realizaron los respectivos análisis.

\subsection{Análisis Fisicoquímicos}

El pH y la acidez expresada en \% ácido cítrico se llevó a acabo de acuerdo al método descrito en la NTC 4592 y 4623, respectivamente. La concentración de vitamina C (mg/100 g de pimentón) en las muestras se determinó considerando el protocolo descrito previamente en el trabajo de Ordoñez-Santos y Vásquez-Riascos (2010). La concentración de clorofila total (mg/100 g de pimentón) en las muestras se cuantifico espectrofotométricamente a $652 \mathrm{~nm}$ en una solución de acetona al $80 \%$ de acuerdo al trabajo de Fernández y Johnston (1986). Todas las evaluaciones fisicoquímicas se realizaron por duplicado.

\subsection{Evaluación del Color Superficial}

Las coordenadas $\mathrm{CIE}_{\mathrm{Lab}}$ en las muestras se determinaron en un colorímetro Minolta CR-400, empleando un iluminante D65, y un observador de $2^{\circ}$. La calibración del equipo se efectuó con la placa de valores de referencia $\mathrm{Y}=89,5 ; \mathrm{x}=0,3176 ; \mathrm{y}=0,3347$, y con los valores colorimétricos a y b se estimó la saturación (C) y el tono (h) de acuerdo a (1) y (2)

$$
\begin{aligned}
& C=\left[\left(a^{* 2}+b^{* 2}\right)\right]^{0,5} \\
& h=\tan ^{-1}\left(b^{*} / a^{*}\right)
\end{aligned}
$$




\subsection{Análisis Estadístico}

El diseño experimental corresponde a un diseño aleatorizado simple de un factor con cuatro tratamientos (fresco, vapor, agua y microondas), se efectuó el respectivo análisis de ANOVA y la prueba de Tukey para identificar las diferencia entre los tratamientos. Todos los análisis se realizaron bajo el software SPSS para Windows v.18.

\section{RESULTADOS Y DISCUSIÓN}

En la Tabla 1 se relacionan los valores medios de los parámetros fisicoquímicos evaluados y su respectivo análisis estadístico. Respecto al material fresco, el pH y la acidez obtenidos en el presente estudio concuerdan con los reportados por Méndez et al. (2004) en materiales verdes de Capsicum sp. (5,930 y 0,158 \%, respectivamente).

Tabla 1. Cambios de las propiedades fisicoquímicas en pimentón verde durante los procesos de cocción. Fuente: Autores

\begin{tabular}{lccccc}
\hline \multicolumn{1}{c}{ Variable } & Fresco & Agua & Vapor & Microondas & ANOVA \\
\hline pH & $5,73 \pm 0,250$ & $5,37 \pm 0,010$ & $5,22 \pm 0,270$ & $5,50 \pm 0,250$ & NS \\
\%Acidez & $0,10 \pm 0,005^{\mathrm{a}}$ & $0,07 \pm 0,001^{\mathrm{c}}$ & $0,08 \pm 0,000^{\mathrm{b}}$ & $0,09 \pm 0,001^{\mathrm{a}}$ & $* *$ \\
Vitamina C $^{1}$ & $147,84 \pm 4,36^{\mathrm{a}}$ & $79,31 \pm 5,44^{\mathrm{b}}$ & $90,10 \pm 1,10^{\mathrm{b}}$ & $139,37 \pm 3,30^{\mathrm{a}}$ & $* * *$ \\
Clorofila $^{1}$ & $3,25 \pm 0,14^{\mathrm{b}}$ & $4,44 \pm 0,04^{\mathrm{a}}$ & $3,47 \pm 0,04^{\mathrm{b}}$ & $2,61 \pm 0,04^{\mathrm{c}}$ & $* * *$ \\
$\mathrm{~L}$ & $32,32 \pm 1,57$ & $34,27 \pm 2,21$ & $35,24 \pm 0,75$ & $38,38 \pm 2,54$ & $\mathrm{NS}$ \\
$\mathrm{a}^{*}$ & $-9,91 \pm 1,43$ & $-10,82 \pm 0,20$ & $-8,68 \pm 1,54$ & $-10,90 \pm 1,65$ & $\mathrm{NS}$ \\
$\mathrm{b}^{*}$ & $10,20 \pm 1,60$ & $12,43 \pm 2,23$ & $13,07 \pm 1,22$ & $10,20 \pm 3,01$ & $\mathrm{NS}$ \\
$\mathrm{C} *$ & $14,22 \pm 2,14$ & $16,51 \pm 1,80$ & $15,75 \pm 0,16$ & $14,94 \pm 3,26$ & $\mathrm{NS}$ \\
$\mathrm{h}$ & $-45,80 \pm 0,35$ & $-48,71 \pm 4,60$ & $-56,37 \pm 7,13$ & $-42,59 \pm 4,23$ & $\mathrm{NS}$
\end{tabular}

${ }^{1} \mathrm{mg} / 100 \mathrm{~g}$ de pimentón, dentro de una fila los valores con diferente letra representa diferencias significativas (Tukey, $\mathrm{p}<0,05$ ) 
En cuanto a la concentración de la vitamina $\mathrm{C}$ en las muestras analizadas (Tabla 1), se confirma que el pimentón fresco es una importante fuente de vitamina $\mathrm{C}$, excede en $87 \mathrm{mg}$ a la Cantidad Diaria Recomendada (CDR) de 60 mg (Carr \& Frei, 1999), y está entre los valores registrados en pimentón verde por otros estudios Rai et al. (2011) y Cao et al. (2012) (35-550 mg vitamina C/100 g de pimentón), mientras que el contenido de clorofila en las muestras evaluadas (Tabla 1), son superadas por los valores obtenidos por Rai et al. (2011) (8 mg/100 g de pimentón). El color superficial (L, $\mathrm{a}, \mathrm{b})$ en el pimentón fresco (Tabla 1), difieren a las registrados por Ornelas-Paz et al. (2013) en pimentón verde ( $\mathrm{L}=60, \mathrm{a}=-20, \mathrm{~b}=40)$. Durante los tratamientos de cocción el pH y las variables de color superficial CIElab no cambian estadísticamente, por el contrario la acidez, la concentración de vitamina $\mathrm{C}$ y la clorofila se ven afectadas significativamente por el tipo de tratamiento térmico (Tabla 1). En este estudio el tratamiento de cocción con microondas no afectó significativamente la acidez y la concentración de vitamina $\mathrm{C}$ en el pimentón verde, por el contrario, los tratamientos de calor con agua $\left(98^{\circ} \mathrm{C}\right)$ y vapor $\left(97^{\circ} \mathrm{C}\right)$ disminuyeron estadísticamente estas dos variables fisicoquímicas, tal como se puede observar en la Tabla 1 y en las Fig. 1 y 2 .

Probablemente la ausencia de procesos de lixiviación en el tratamiento de cocción con microonda permitió retener una mayor concentración de este antioxidante en las muestras evaluadas, otros estudios reportan pérdidas significativas de vitamina $\mathrm{C}$ en pimentón verde durante los tratamientos de cocción (Castro et al., 2008; Chuah et al., 2008; Castro et al., 2011; Ornelas-Paz et al., 2013). La degradación de esta importante molécula se debe, principalmente, a las reacciones químicas que involucran la oxidación a la forma de hidroascórbico (DHAA), hidrólisis del DHAA al ácido 2,3-dicetogulonico y la generación por polimerización de productos inactivos nutricionalmente (Chuah et al., 2008). En el trabajo realizado por Ornelas-Paz et al. (2013) informan que las pérdidas de vitamina $\mathrm{C}$ en el pimentón verde durante los tratamientos de cocción puede ser consecuencia de la baja concentración de otros antioxidantes como los carotenoides, compuestos fenólicos, capsinoides y tocoferol, ya que estos compuestos pueden actuar directamente o indirectamente en la oxidación de la vitamina $\mathrm{C}$. 


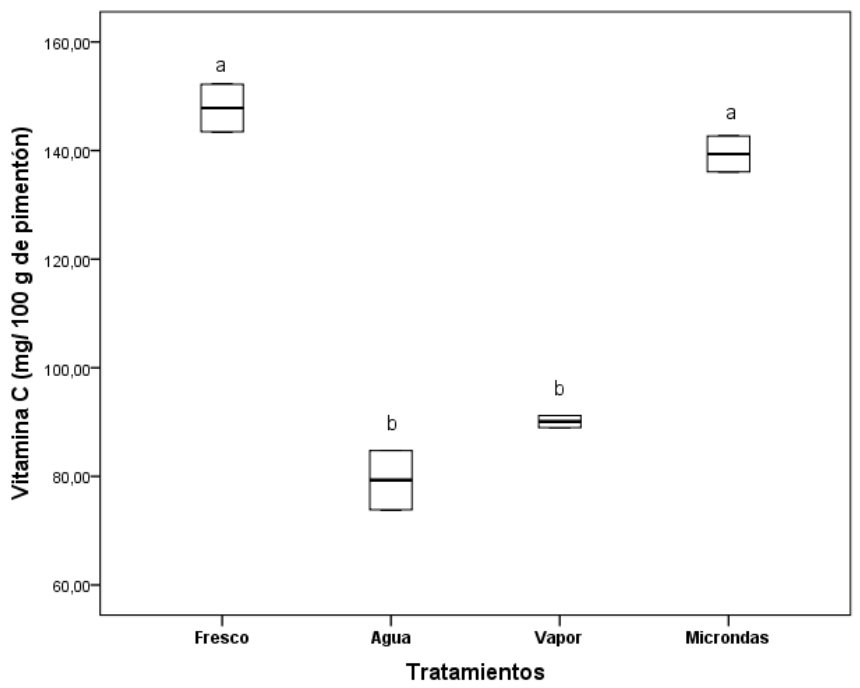

Fig. 1. Cambios en la concentración de vitamina $\mathrm{C}$ en pimentón verde durante los tratamientos de cocción. Fuente: Autores

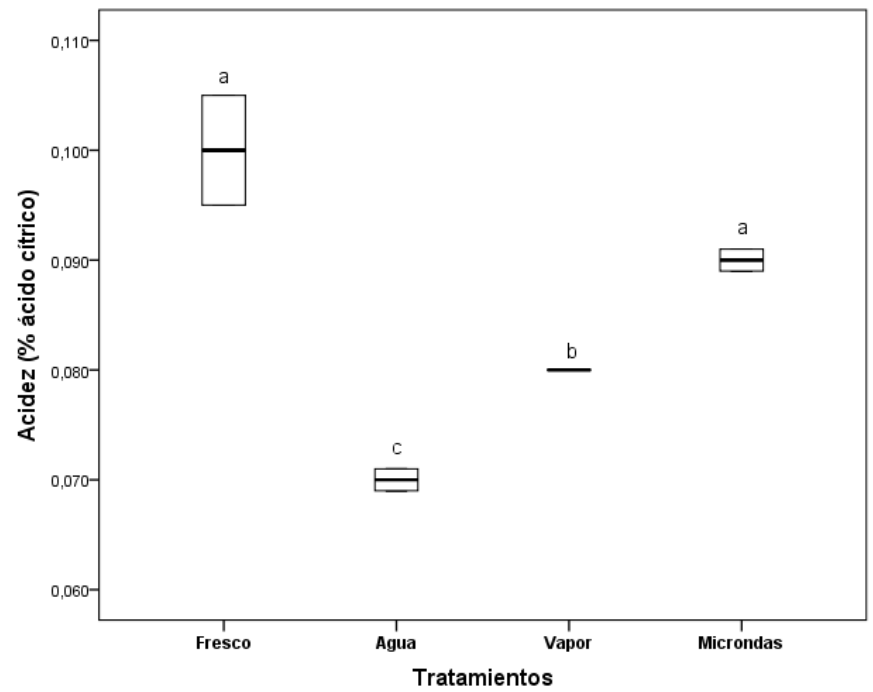

Fig. 2. Cambios en el porcentaje de acidez en pimentón verde durante los tratamientos de cocción. Fuente: Autores 
Como se puede observar en la Tabla 1 y la Fig. 3, el método de cocción por microondas reduce significativamente la concentración de clorofila, mientras que el tratamiento con agua aumenta estadísticamente el contenido de este pigmento y el tratamiento con vapor de agua no afectó significativamente las muestras evaluadas.

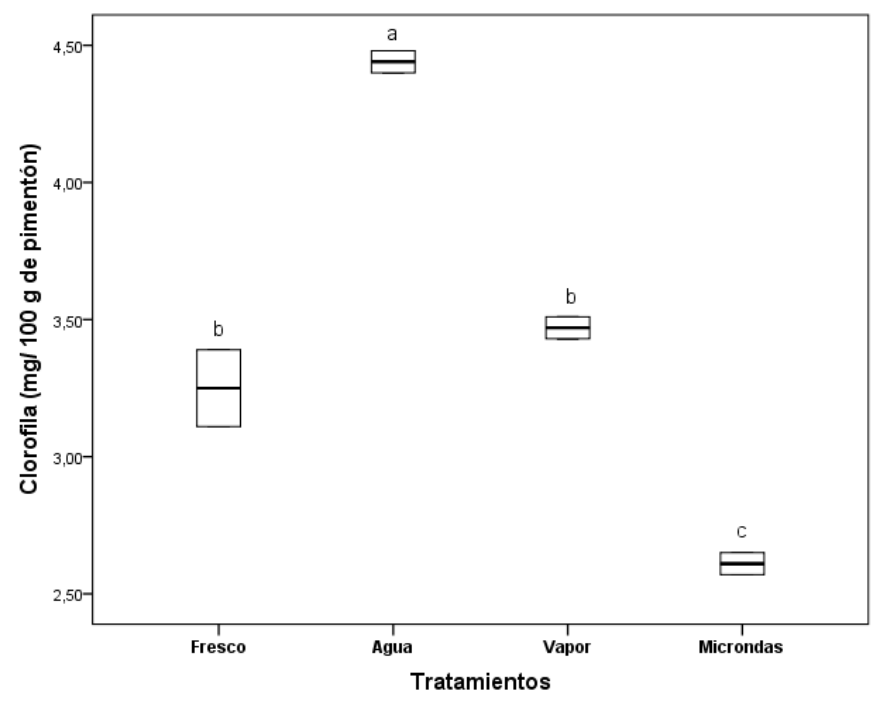

Fig. 3. Cambios en la concentración de clorofila en pimentón verde durante los tratamientos de cocción. Fuente: Autores

La reducción de la clorofila durante la cocción de vegetales verdes ha sido reportada previamente por Martínez-Hernández et al. (2013) y Martínez et al. (2013), los autores afirman que la degradación durante los métodos de cocción, probablemente es consecuencia de la ruptura irreversible de la clorofila a y b en sus derivados principales feofitina a y b. El aumento aparente de la clorofila en el tratamiento con agua probablemente es consecuencia de fenómenos de lixiviación en las muestras analizadas que permitió concentrar este pigmento. Los cambios significativos en la concentración de la clorofila durante los tratamientos de cocción no evidenciaron pérdidas significativas en los atributos de color CIE $_{\text {Lab }}$ en las muestras evaluadas, resultado mostrado en la Tabla 
1 , los cuales señalan que el color superficial del pimentón verde se conserva después de los métodos de cocción. Contrario a nuestros resultados Ornelas-Paz et al. (2010) y Ornelas-Paz et al. (2013) si reportan un efecto significativo de los procesos de cocción sobre el color superficial $\mathrm{CIE}_{\mathrm{Lab}}$ en pimentones verdes.

\section{CONCLUSIONES}

En el presente trabajo se puede concluir que el pimentón verde es una fuente importante de vitamina $\mathrm{C}$ capaz de cubrir las necesidades mínimas diarias recomendadas de este antioxidante en la alimentación humana, y el método de cocción por microondas es el proceso que logra el mayor nivel de retención de este antioxidante. Por otra parte, a pesar de que el método de cocción por microondas degrade la clorofila en feofitina a y b, el color superficial del pimentón verde no logra ser afectado después de la cocción.

\section{REFERENCIAS}

Cao, S., Yang, Z., Zheng, Y. (2012). Effect of 1-methylcyclopene on senescence and quality maintenance of green bell pepper fruit during storage at $20 \circ \mathrm{C}$. Postharvest Biology and Technology, 70, 1-6.

Carr, A.C., Frei, B. (1999). Toward a new recommended dietary allowance for vitamin $\mathrm{C}$ based on antioxidant and health effects in humans. American Journal of Clinical Nutrition, 69(6), 1086-1107.

Castro, S.M., et al., (2008). Effect of thermal blanching and of high pressure treatments on sweet green and red bell pepper fruits (Capsicum annuum L.). Food Chemistry, 107(4), 1436-1449.

Castro, S.M., Saraiva, J.A., Fernando M.J., Delgadillo, D.I. (2011). Effect of mild pressure treatments and thermal blanching on yellow bell peppers (Capsicum annuum L.). Food Science and Technology, 44(2), 363-369.

Chuah, A.M., Lee, Y.C., Yamaguchi, T., Takamura, H., Yin, L.J., Matoba, T. (2008). Effect of cooking on the antioxidant properties of coloured peppers. Food Chemistry, 111(1), 20-28.

Fernández, M., Johnston, M. (1986). Fisiología vegetal experimental. $1^{\mathrm{a}}$ edición, 19-27, Instituto Interamericano de Cooperación para la Agricultura (IICA), San José de Costa Rica. 
Ghasemnezhad, M., Mohamad, S., Gholam, A.P. (2011). Variation in phenolic compounds, ascorbic acid and antioxidant activity of five coloured bell pepper (Capsicum annum) fruits at two different harvest times. Journal of Functional Foods, 3(1), 44-49.

NTC 4592. (1999). Productos de frutas y verduras. Determinación del pH. Bogotá: Instituto Colombiano de Normas Técnicas y Certificación ICONTEC.

NTC 4623. (1999). Productos de frutas y verduras: Determinación de la acidez titulable. Bogotá: Instituto Colombiano de Normas Técnicas y Certificación - ICONTEC.

Martínez, S., Pérez, N., Carballo, J., Franco, I. (2013). Effect of blanching methods and frozen storage on some quality parameters of turnip greens ("grelos"), LWT. Food Science and Technology, 51(1), 383-392.

Martínez-Hernández, G.B., Artés-Hernández, F., Gómez, P.A., Artés, F. (2013). Quality changes after vacuum-based and conventional industrial cooking of kailan-hybrid broccoli throughout retail cold storage, LWT. Food Science and Technology 50(2), 707-714.

Méndez, M.A., Ligarreto, G.A., Hernández, M.A., Melgarejo, L.M. (2004). Evaluating growth and determining harvesting index in four types of hot chilli pepper (Capsicum sp.) grown in the Colombian Amazonian region. Agronomía Colombiana, 22(1), 7-17.

Ordoñez-Santos, L.E., Vásquez-Riascos, A. (2010). Effect of processing and storage time on the vitamin $\mathrm{C}$ and lycopene contents of nectar of pink guava (Psidium guajava L.). Archivos Latinoamericanos de $\mathrm{Nu}$ trición, 60(3), 280-284.

Ornelas-Paz, J.J. et al. (2010). Effect of cooking on the capsaicinoids and phenolics contents of Mexican peppers. Food Chemistry, 119(4), 16191625.

Ornelas-Paz, J.J., et al., (2013). Effect of heat treatment on the content of some bioactive compounds and free radical-scavenging activity in pungent and non-pungent peppers. Food Research International, 50(2), 519-525.

Rai, D.R., Kaur, P., Patil, R.T. (2011). Quality changes in fresh-cut capsicum (Capsicum annuum) shreds under modified atmospheres during simulated retail and home storage. Journal of Food Processing and Preservation, 35(4), 402-409.

Zhuang, Y., Chen, L., Sun, L., Cao, J. (2012). Bioactive characteristics and antioxidant activities of nine peppers. Journal of Functional Foods, 4(1), 331-338. 